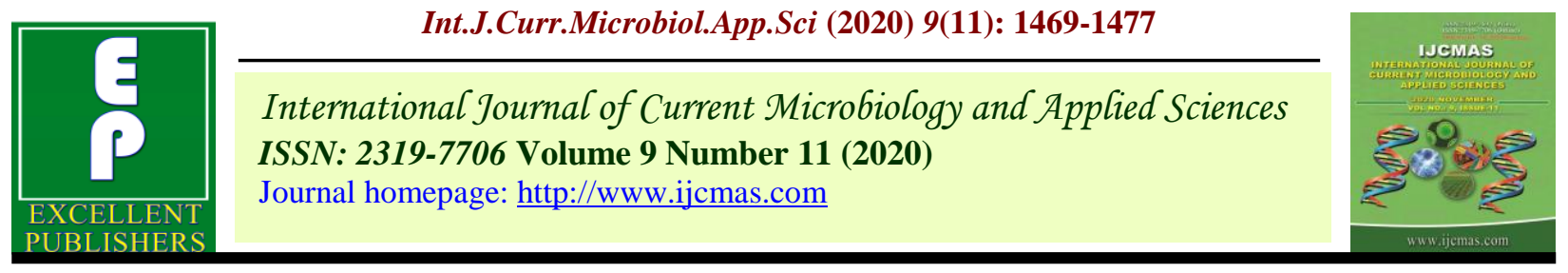

Original Research Article

https://doi.org/10.20546/ijcmas.2020.911.173

\title{
Fitting Crop Production in Northern Zone of Kerala during El Niño Conditions
}

\author{
R. K. Rajesh Krishnan ${ }^{*}$ and P. K. Retheesh \\ Regional Agricultural Research Station, Kasargod, Kerala, India \\ *Corresponding author
}

\section{A B S T R A C T}

\section{Keywords \\ El Niño conditions, Fitting crop production}

Article Info

Accepted:

12 October 2020

Available Online:

10 November 2020
Weather data collected from the agromet observatory located at Regional Agricultural research station, Kasargod was used for markov chain analysis to mark the weekly wet and dry spell distribution in the region during the climatic conditions El Nino and La Nina. Weather cock software and manual calculation was done to arrive at the results. Both initial and conditional probability was found out for annual, SW Monsoon and NE Monsoon weeks based on the methodology. The length of growing period was also calculated based on this for three climatic situations based on the method suggested by FAO, El nino conditions possess better LGP (31 weeks) as compared with Lanina and neutral years.

\section{Introduction}

Rainfall is a unique weather variable which shows higher degrees of variation in its spatial and temporal distribution over earth's surface, Since the land use pattern and vegetation over a region is largely influenced by rainfall, its spatial distribution over the growing period of crops play a vital role in the success and failure of crops. Water excess as well as less than the crop requirement has effect on the crop growth. Hence study on identifying the continuous wet spell and dry spell can aid in identifying the successful crop-growing period of a region within a year. Even though there are many methods which can predict the dry and wet spells Markov chain analysis is a better methodology to predict these in terms of probability.

The information on occurrence of dry and wet spell can apparently provide an idea about the crop growing period on weekly basis and planning agricultural operations like sowing, weeding, irrigation etc on rainfed farming areas. Hence markov chain analysis can be used as a best option with limited data available areas to calculate the wet and dry spell probability where precipitation and evaporation data is available. It can be used as a decision support tool in farm planning for crop production within the growing season. Indian agriculture is directly related with the monsoon and hence performance of monsoon 
greatly impacts cropping pattern and food production of the nation. But most often, it is the quantity of the monsoon rainfall is used for estimating the impact of monsoon. In reality it is the distribution of rainfall within the year along with quantum decides the farming operations. Very few attempts have been made to analyse the rainfall effects on the basis of wet and dry spells to arrive at the crop growing decisions. In general, it is reviewed that there will be decrease in rainfall for SW monsoon and increase in rainfall during NE monsoon for peninsular region during El Niño years and vice versa for La Niña years. Markov chain tool is implicated here to find out the distribution of wet spells during El Niño and La Niña years compared with neutral years so as to find the maximum possible wet period which helps in identifying maximum cropping duration for respective periods.

\section{Materials and Methods}

Weather data collected from the agromet observatory located at Regional Agricultural research station, Pilicode was used for this markov analysis. The coordinates of the observatory are $11.2^{\circ} \mathrm{N}, 77.5^{\circ} \mathrm{E}, 13 \mathrm{MSL}$. Weekly rainfall and evaporation data for 52 standard weeks were collected from 1961 to 2018 and the El Niño, La Niña and neutral years were found out based on information provided by NOAA and years were classified accordingly (Table 1).

Markov chain analysis was implicated as mentioned by Veeraputhiran et al., to categorize wet and dry weeks. Weather cock software and manual calculation was done to arrive at the results. Based on the evaporation data in Kasaragod, wet spells are those weeks where annual weekly precipitation exceeds the weekly evaporation. Here it was $27 \mathrm{~mm}$. Hence wet weeks were ones with precipitation more than $27 \mathrm{~mm}$ and dry weeks with precipitation less than $27 \mathrm{~mm}$. Markov chain analysis was done for annual weekly (1 to 52 weeks), SW monsoon (22 to 39 weeks) and NW monsoon (40 to 52 weeks) as defined by IMD and it was separately done for all the climatic situations of El Niño, Lanina and neutral years. Both initial and conditional probability was found out for the above mentioned periods based on the following methodology.

\section{a Initial Probability}

$\mathrm{P}(\mathrm{W})=\mathrm{n}(\mathrm{W}) / \mathrm{n}(\mathrm{W}+\mathrm{D}) * 100$

$\mathrm{P}(\mathrm{D})=\mathrm{n}(\mathrm{D}) / \mathrm{n}(\mathrm{D}+\mathrm{W}) * 100$

P(W): Probability of the week being wet

P(D): Probability of the week being dry

$\mathrm{n}(\mathrm{W})$ : Frequency of wet weeks

n(D): Frequency of dry weeks

$n(D+W)$ : Total no. of weeks

\section{b. Conditional Probability}

$\mathrm{P}(\mathrm{W} / \mathrm{D})=\mathrm{n}(\mathrm{W} / \mathrm{D}) / \mathrm{nD} * 100$

$\mathrm{P}(\mathrm{D} / \mathrm{D})=\mathrm{n}(\mathrm{D} / \mathrm{D}) / \mathrm{nD} * 100$

$\mathrm{P}(\mathrm{W} / \mathrm{W})=\mathrm{n}(\mathrm{W} / \mathrm{W}) / \mathrm{nW}^{*} 100$

$\mathrm{P}(\mathrm{D} / \mathrm{W})=\mathrm{n}(\mathrm{D} 2 \mathrm{~W} 1) / \mathrm{nW}^{*} 100$

P (W/D): Probability of a week being wet after a week being dry

P (D/D): Probability of a week being dry after a week being dry

P (W/W): Probability of a week being wet after a week being wet

P (D2/W1): Probability of a week being dry after a week being wet

$\mathrm{N}$ (W/D): Frequency of wet week after dry week

n (D/D): Frequency of dry week after dry week 
n (W/W): Frequency of wet week after wet week

n (D/W): Frequency of dry week after wet week

n D: Total frequency of dry weeks

$\mathrm{n} \mathrm{W}$ : Total frequency of wet weeks

\section{Results and Discussion}

The results obtained from the analysis are presented in Table 2 and 3. Table 2 provides the initial probability of occurance of wet and dry spells for 52 standard weeks individually.

\section{Initial probability}

From the table 3 it can be inferred that during all the three climatic situations there is very little probability is there for wet spells during the first 14 weeks of respective years, expect for neutral years where minor probability is there at the start of summer season. But during the end weeks of summer season, Neutral years showing better probability of wet spell than other climatic situations.

The probability is higher when it approaches the monsoon season in all climatic situations. During the SW monsoon period, all of the situations do not exert much impact on the wet spell period and the probability remains around 0.9 except at the end of the season.

During NE monsoon period, even though neutral situation showing some better probability at the start of the season, El Niño conditions provide higher probability for rest of the season.

Table.1 Various El Niño, Lanina and neutral years

\begin{tabular}{|c|c|c|}
\hline El nino & La Niña & Neutral \\
\hline 1969 & 1964 & 1961 \\
\hline 1976 & 1971 & 1962 \\
\hline 1977 & 1974 & 1966 \\
\hline 1979 & 1983 & 1967 \\
\hline 2004 & 1984 & 1978 \\
\hline 2006 & 2000 & 1980 \\
\hline 2014 & 2005 & 1981 \\
\hline 1963 & 2008 & 1985 \\
\hline 1968 & 2016 & 1989 \\
\hline 1986 & 2017 & 1990 \\
\hline 1994 & 1970 & 1992 \\
\hline 2002 & 1995 & 1993 \\
\hline 2009 & 2011 & 1996 \\
\hline 1965 & 1973 & 2001 \\
\hline 1972 & 1975 & 2003 \\
\hline 1987 & 1988 & 2012 \\
\hline 1991 & 1998 & 2013 \\
\hline 1982 & 1999 & \\
\hline 1997 & 2007 & \\
\hline 2015 & 2010 & \\
\hline 2018 & & \\
\hline
\end{tabular}


Table.2 Frequency analysis on wet spells and dry spells during Annual rainfall, SW monsoon and NE Monsoon

\begin{tabular}{|c|c|c|c|c|c|c|c|c|c|}
\hline & \multicolumn{3}{|c|}{ Annual } & \multicolumn{3}{|c|}{ SW Monsoon } & \multicolumn{3}{|c|}{ NE Monsoon } \\
\hline Probability & El Nino & La Niña & Neutral & El Nino & La Niña & Neutral & El Nino & La Niña & Neutral \\
\hline $\mathbf{P}(\mathbf{W})$ & 27 & 23 & 25 & 17 & 17 & 17 & 6 & 4 & 5 \\
\hline $\mathbf{P}(\mathrm{D})$ & 25 & 29 & 27 & 0 & 0 & 0 & 7 & 9 & 8 \\
\hline $\mathbf{P}(\mathbf{W} / \mathbf{W})$ & 24 & 22 & 24 & 17 & 17 & 17 & 4 & 4 & 5 \\
\hline $\mathbf{P}(\mathrm{D} / \mathrm{D})$ & 22 & 28 & 26 & 0 & 0 & 0 & 4 & 8 & 7 \\
\hline $\mathbf{P}(\mathrm{W} / \mathrm{D})$ & 3 & 1 & 1 & 0 & 0 & 0 & 2 & 0 & 0 \\
\hline $\mathbf{P}(\mathrm{D} / \mathrm{W})$ & 3 & 1 & 1 & 0 & 0 & 0 & 3 & 1 & 1 \\
\hline LGP & \multicolumn{3}{|c|}{$\begin{array}{l}\text { El Nino } \\
\text { May })-49\left(09^{\text {th }} \text { Dec }\right) \\
31 \text { weeks }\end{array}$} & \multicolumn{3}{|c|}{$\begin{array}{l}\text { La Niña } \\
\text { May) }-47\left(25^{\text {th }} \text { Nov }\right) \\
27 \text { weeks }\end{array}$} & \multicolumn{3}{|c|}{$\begin{array}{l}\text { Neutral } \\
\text { May)- } 49\left(09^{\text {th }} \text { Dec }\right) \\
29 \text { weeks }\end{array}$} \\
\hline
\end{tabular}

Table.3 Conditional probability for 52 Meteorological Standard weeks

\begin{tabular}{|c|c|c|c|c|c|c|c|c|c|c|c|c|}
\hline & \multicolumn{3}{|c|}{$\mathrm{P}(\mathrm{W} / \mathrm{W})$} & \multicolumn{3}{|c|}{$\mathrm{P}(\mathrm{D} / \mathrm{W})$} & \multicolumn{3}{|c|}{$\mathrm{P}(\mathrm{D} / \mathrm{D})$} & \multicolumn{3}{|c|}{$\mathrm{P}(\mathrm{W} / \mathrm{D})$} \\
\hline $\begin{array}{l}\text { WE } \\
\text { EK }\end{array}$ & $\begin{array}{l}\text { El } \\
\text { Niño }\end{array}$ & $\begin{array}{l}\text { La } \\
\text { Niña }\end{array}$ & $\begin{array}{l}\text { Neut } \\
\text { rel }\end{array}$ & $\begin{array}{l}\text { El } \\
\text { Niño }\end{array}$ & $\begin{array}{l}\text { La } \\
\text { Niña }\end{array}$ & $\begin{array}{l}\text { Neut } \\
\text { ral }\end{array}$ & $\begin{array}{l}\text { El } \\
\text { Niño }\end{array}$ & $\begin{array}{l}\text { La } \\
\text { Niña }\end{array}$ & $\begin{array}{l}\text { Neut } \\
\text { ral }\end{array}$ & $\begin{array}{l}\text { EI } \\
\text { Niño }\end{array}$ & $\begin{array}{l}\text { La } \\
\text { Niña }\end{array}$ & $\begin{array}{l}\text { Neut } \\
\text { ral }\end{array}$ \\
\hline 1 & 0.00 & 0.00 & 0.00 & 0.00 & 0.00 & 1.00 & 1.00 & 1.00 & 0.93 & 0.00 & 0.00 & 0.07 \\
\hline 2 & 0.00 & 0.00 & 0.00 & 0.00 & 0.00 & 1.00 & 1.00 & 0.95 & 1.00 & 0.00 & 0.05 & 0.00 \\
\hline 3 & 0.00 & 0.00 & 0.00 & 0.00 & 1.00 & 0.00 & 1.00 & 1.00 & 1.00 & 0.00 & 0.00 & 0.00 \\
\hline 4 & 0.00 & 0.00 & 0.00 & 0.00 & 0.00 & 0.00 & 1.00 & 1.00 & 1.00 & 0.00 & 0.00 & 0.00 \\
\hline 5 & 0.00 & 0.00 & 0.00 & 0.00 & 0.00 & 0.00 & 1.00 & 1.00 & 1.00 & 0.00 & 0.00 & 0.00 \\
\hline 6 & 0.00 & 0.00 & 0.00 & 0.00 & 0.00 & 0.00 & 1.00 & 1.00 & 1.00 & 0.00 & 0.00 & 0.00 \\
\hline 7 & 0.00 & 0.00 & 0.00 & 0.00 & 0.00 & 0.00 & 1.00 & 1.00 & 1.00 & 0.00 & 0.00 & 0.00 \\
\hline 8 & 0.00 & 0.00 & 0.00 & 0.00 & 0.00 & 0.00 & 1.00 & 1.00 & 1.00 & 0.00 & 0.00 & 0.00 \\
\hline 9 & 0.00 & 0.00 & 0.00 & 0.00 & 0.00 & 0.00 & 1.00 & 0.95 & 1.00 & 0.00 & 0.05 & 0.00 \\
\hline 10 & 0.00 & 1.00 & 0.00 & 0.00 & 0.00 & 0.00 & 1.00 & 0.95 & 1.00 & 0.00 & 0.05 & 0.00 \\
\hline 11 & 0.00 & 0.00 & 0.00 & 0.00 & 1.00 & 0.00 & 1.00 & 1.00 & 1.00 & 0.00 & 0.00 & 0.00 \\
\hline 12 & 0.00 & 0.00 & 0.00 & 0.00 & 0.00 & 0.00 & 1.00 & 0.95 & 1.00 & 0.00 & 0.05 & 0.00 \\
\hline 13 & 0.00 & 0.00 & 0.00 & 0.00 & 1.00 & 0.00 & 1.00 & 1.00 & 0.94 & 0.00 & 0.00 & 0.06 \\
\hline 14 & 0.00 & 0.00 & 0.00 & 0.00 & 0.00 & 1.00 & 0.95 & 0.95 & 0.94 & 0.05 & 0.05 & 0.06 \\
\hline 15 & 0.00 & 0.00 & 1.00 & 1.00 & 1.00 & 0.00 & 0.89 & 0.84 & 1.00 & 0.11 & 0.16 & 0.00 \\
\hline 16 & 0.50 & 0.33 & 0.00 & 0.50 & 0.67 & 1.00 & 0.83 & 0.94 & 0.94 & 0.17 & 0.06 & 0.06 \\
\hline 17 & 0.25 & 0.00 & 0.00 & 0.75 & 1.00 & 1.00 & 0.94 & 0.83 & 0.75 & 0.06 & 0.17 & 0.25 \\
\hline 18 & 0.00 & 0.33 & 0.50 & 1.00 & 0.67 & 0.50 & 0.72 & 0.88 & 0.85 & 0.28 & 0.12 & 0.15 \\
\hline 19 & 0.60 & 0.33 & 0.25 & 0.40 & 0.67 & 0.75 & 0.80 & 0.88 & 0.62 & 0.20 & 0.12 & 0.38 \\
\hline 20 & 0.50 & 0.67 & 0.67 & 0.50 & 0.33 & 0.33 & 0.79 & 0.71 & 0.55 & 0.21 & 0.29 & 0.45 \\
\hline 21 & 0.33 & 0.86 & 0.56 & 0.67 & 0.14 & 0.44 & 0.71 & 0.62 & 0.88 & 0.29 & 0.38 & 0.13 \\
\hline 22 & 0.83 & 0.91 & 1.00 & 0.17 & 0.09 & 0.00 & 0.50 & 0.44 & 0.36 & 0.50 & 0.56 & 0.64 \\
\hline 23 & 1.00 & 1.00 & 1.00 & 0.00 & 0.00 & 0.00 & 0.25 & 0.20 & 0.00 & 0.75 & 0.80 & 1.00 \\
\hline 24 & 0.94 & 1.00 & 1.00 & 0.06 & 0.00 & 0.00 & 0.50 & 0.00 & 0.00 & 0.50 & 1.00 & 0.00 \\
\hline 25 & 1.00 & 1.00 & 1.00 & 0.00 & 0.00 & 0.00 & 0.00 & 0.00 & 0.00 & 1.00 & 0.00 & 0.00 \\
\hline
\end{tabular}




\begin{tabular}{|c|c|c|c|c|c|c|c|c|c|c|c|c|}
\hline 26 & 1.00 & 1.00 & 0.94 & 0.00 & 0.00 & 0.06 & 0.00 & 0.00 & 0.00 & 0.00 & 0.00 & 0.00 \\
\hline 27 & 0.95 & 1.00 & 0.94 & 0.05 & 0.00 & 0.06 & 0.00 & 0.00 & 0.00 & 0.00 & 0.00 & 1.00 \\
\hline 28 & 1.00 & 1.00 & 1.00 & 0.00 & 0.00 & 0.00 & 0.00 & 0.00 & 0.00 & 1.00 & 0.00 & 1.00 \\
\hline 29 & 1.00 & 0.95 & 1.00 & 0.00 & 0.05 & 0.00 & 0.00 & 0.00 & 0.00 & 0.00 & 0.00 & 0.00 \\
\hline 30 & 0.90 & 0.89 & 1.00 & 0.10 & 0.11 & 0.00 & 0.00 & 0.00 & 0.00 & 0.00 & 1.00 & 0.00 \\
\hline 31 & 1.00 & 0.94 & 0.94 & 0.00 & 0.06 & 0.06 & 0.00 & 0.50 & 0.00 & 1.00 & 0.50 & 0.00 \\
\hline 32 & 0.90 & 1.00 & 1.00 & 0.10 & 0.00 & 0.00 & 0.00 & 0.00 & 0.00 & 0.00 & 1.00 & 1.00 \\
\hline 33 & 0.89 & 0.95 & 0.94 & 0.11 & 0.05 & 0.06 & 0.00 & 0.00 & 0.00 & 1.00 & 0.00 & 0.00 \\
\hline 34 & 0.78 & 0.79 & 0.88 & 0.22 & 0.21 & 0.13 & 0.00 & 0.00 & 1.00 & 1.00 & 1.00 & 0.00 \\
\hline 35 & 0.75 & 0.94 & 0.71 & 0.25 & 0.06 & 0.29 & 0.75 & 0.50 & 0.33 & 0.25 & 0.50 & 0.67 \\
\hline 36 & 0.69 & 0.71 & 0.67 & 0.31 & 0.29 & 0.33 & 0.43 & 0.33 & 0.60 & 0.57 & 0.67 & 0.40 \\
\hline 37 & 0.69 & 0.79 & 0.70 & 0.31 & 0.21 & 0.30 & 0.57 & 0.50 & 0.57 & 0.43 & 0.50 & 0.43 \\
\hline 38 & 0.33 & 0.86 & 0.60 & 0.67 & 0.14 & 0.40 & 0.13 & 0.83 & 0.71 & 0.88 & 0.17 & 0.29 \\
\hline 39 & 0.45 & 0.46 & 0.88 & 0.55 & 0.54 & 0.13 & 0.56 & 0.57 & 0.44 & 0.44 & 0.43 & 0.56 \\
\hline 40 & 0.56 & 0.67 & 0.75 & 0.44 & 0.33 & 0.25 & 0.73 & 0.64 & 0.40 & 0.27 & 0.36 & 0.60 \\
\hline 41 & 0.25 & 0.70 & 0.83 & 0.75 & 0.30 & 0.17 & 0.50 & 0.70 & 0.60 & 0.50 & 0.30 & 0.40 \\
\hline 42 & 0.75 & 0.70 & 0.67 & 0.25 & 0.30 & 0.33 & 0.42 & 0.60 & 0.80 & 0.58 & 0.40 & 0.20 \\
\hline 43 & 0.38 & 0.64 & 0.67 & 0.62 & 0.36 & 0.33 & 0.71 & 0.78 & 0.50 & 0.29 & 0.22 & 0.50 \\
\hline 44 & 0.29 & 0.56 & 0.40 & 0.71 & 0.44 & 0.60 & 0.54 & 0.82 & 0.71 & 0.46 & 0.18 & 0.29 \\
\hline 45 & 0.38 & 0.43 & 0.33 & 0.63 & 0.57 & 0.67 & 0.75 & 0.69 & 0.91 & 0.25 & 0.31 & 0.09 \\
\hline 46 & 0.67 & 0.14 & 0.00 & 0.33 & 0.86 & 1.00 & 1.00 & 0.92 & 0.64 & 0.00 & 0.08 & 0.36 \\
\hline 47 & 0.25 & 0.00 & 0.20 & 0.75 & 1.00 & 0.80 & 0.69 & 0.94 & 1.00 & 0.31 & 0.06 & 0.00 \\
\hline 48 & 0.00 & 1.00 & 0.00 & 1.00 & 0.00 & 1.00 & 1.00 & 0.89 & 0.94 & 0.00 & 0.11 & 0.06 \\
\hline 49 & 0.00 & 0.00 & 1.00 & 0.00 & 1.00 & 0.00 & 0.90 & 1.00 & 0.94 & 0.10 & 0.00 & 0.06 \\
\hline 50 & 0.00 & 0.00 & 0.00 & 1.00 & 0.00 & 1.00 & 0.83 & 0.95 & 0.93 & 0.17 & 0.05 & 0.07 \\
\hline 51 & 0.00 & 0.00 & 0.00 & 1.00 & 1.00 & 1.00 & 1.00 & 0.95 & 1.00 & 0.00 & 0.05 & 0.00 \\
\hline 52 & 0.00 & 0.00 & 0.00 & 0.00 & 1.00 & 0.00 & 1.00 & 1.00 & 0.94 & 0.00 & 0.00 & 0.06 \\
\hline
\end{tabular}

Table.4 Initial probability for 52 Meteorological Standard weeks

\begin{tabular}{|c|r|r|r|r|r|r|}
\hline & \multicolumn{3}{|c|}{$\mathbf{P}(\mathbf{W})$} & \multicolumn{3}{|c|}{ P(D) } \\
\hline WEEK & El Niño & La Niña & Neutral & El Niño & La Niña & Neutral \\
\hline $\mathbf{1}$ & 0.00 & 0.00 & 0.06 & 1.00 & 1.00 & 0.94 \\
\hline $\mathbf{2}$ & 0.00 & 0.05 & 0.00 & 1.00 & 0.95 & 1.00 \\
\hline $\mathbf{3}$ & 0.00 & 0.00 & 0.00 & 1.00 & 1.00 & 1.00 \\
\hline $\mathbf{4}$ & 0.00 & 0.00 & 0.00 & 1.00 & 1.00 & 1.00 \\
\hline $\mathbf{5}$ & 0.00 & 0.00 & 0.00 & 1.00 & 1.00 & 1.00 \\
\hline $\mathbf{6}$ & 0.00 & 0.00 & 0.00 & 1.00 & 1.00 & 1.00 \\
\hline $\mathbf{7}$ & 0.00 & 0.00 & 0.00 & 1.00 & 1.00 & 1.00 \\
\hline $\mathbf{8}$ & 0.00 & 0.00 & 0.00 & 1.00 & 1.00 & 1.00 \\
\hline $\mathbf{9}$ & 0.00 & 0.05 & 0.00 & 1.00 & 0.95 & 1.00 \\
\hline $\mathbf{1 0}$ & 0.00 & 0.10 & 0.00 & 1.00 & 0.90 & 1.00 \\
\hline $\mathbf{1 1}$ & 0.00 & 0.00 & 0.00 & 1.00 & 1.00 & 1.00 \\
\hline
\end{tabular}




\begin{tabular}{|l|l|l|l|l|l|l|}
\hline $\mathbf{1 2}$ & 0.00 & 0.05 & 0.00 & 1.00 & 0.95 & 1.00 \\
\hline $\mathbf{1 3}$ & 0.00 & 0.00 & 0.06 & 1.00 & 1.00 & 0.94 \\
\hline $\mathbf{1 4}$ & 0.05 & 0.05 & 0.06 & 0.95 & 0.95 & 0.94 \\
\hline $\mathbf{1 5}$ & 0.10 & 0.15 & 0.06 & 0.90 & 0.85 & 0.94 \\
\hline $\mathbf{1 6}$ & 0.20 & 0.10 & 0.06 & 0.80 & 0.90 & 0.94 \\
\hline $\mathbf{1 7}$ & 0.10 & 0.15 & 0.24 & 0.90 & 0.85 & 0.76 \\
\hline $\mathbf{1 8}$ & 0.25 & 0.15 & 0.24 & 0.75 & 0.85 & 0.76 \\
\hline $\mathbf{1 9}$ & 0.30 & 0.15 & 0.35 & 0.70 & 0.85 & 0.65 \\
\hline $\mathbf{2 0}$ & 0.30 & 0.35 & 0.53 & 0.70 & 0.65 & 0.47 \\
\hline $\mathbf{2 1}$ & 0.30 & 0.55 & 0.35 & 0.70 & 0.45 & 0.65 \\
\hline $\mathbf{2 2}$ & 0.60 & 0.75 & 0.76 & 0.40 & 0.25 & 0.24 \\
\hline $\mathbf{2 3}$ & 0.90 & 0.95 & 1.00 & 0.10 & 0.05 & 0.00 \\
\hline $\mathbf{2 4}$ & 0.90 & 1.00 & 1.00 & 0.10 & 0.00 & 0.00 \\
\hline $\mathbf{2 5}$ & 1.00 & 1.00 & 1.00 & 0.00 & 0.00 & 0.00 \\
\hline $\mathbf{2 6}$ & 1.00 & 1.00 & 0.94 & 0.00 & 0.00 & 0.06 \\
\hline $\mathbf{2 7}$ & 0.95 & 1.00 & 0.94 & 0.05 & 0.00 & 0.06 \\
\hline $\mathbf{2 8}$ & 1.00 & 1.00 & 1.00 & 0.00 & 0.00 & 0.00 \\
\hline $\mathbf{2 9}$ & 1.00 & 0.95 & 1.00 & 0.00 & 0.05 & 0.00 \\
\hline $\mathbf{3 0}$ & 0.90 & 0.90 & 1.00 & 0.10 & 0.10 & 0.00 \\
\hline $\mathbf{3 1}$ & 1.00 & 0.90 & 0.94 & 0.00 & 0.10 & 0.06 \\
\hline $\mathbf{3 2}$ & 0.90 & 1.00 & 1.00 & 0.10 & 0.00 & 0.00 \\
\hline $\mathbf{3 3}$ & 0.90 & 0.95 & 0.94 & 0.10 & 0.05 & 0.06 \\
\hline $\mathbf{3 4}$ & 0.80 & 0.80 & 0.82 & 0.20 & 0.20 & 0.18 \\
\hline $\mathbf{3 5}$ & 0.65 & 0.85 & 0.71 & 0.35 & 0.15 & 0.29 \\
\hline $\mathbf{3 6}$ & 0.65 & 0.70 & 0.59 & 0.35 & 0.30 & 0.41 \\
\hline $\mathbf{3 7}$ & 0.60 & 0.70 & 0.59 & 0.40 & 0.30 & 0.41 \\
\hline $\mathbf{3 8}$ & 0.55 & 0.65 & 0.47 & 0.45 & 0.35 & 0.53 \\
\hline $\mathbf{3 9}$ & 0.45 & 0.45 & 0.71 & 0.55 & 0.55 & 0.29 \\
\hline $\mathbf{4 0}$ & 0.40 & 0.50 & 0.71 & 0.60 & 0.50 & 0.29 \\
\hline $\mathbf{4 1}$ & 0.40 & 0.50 & 0.71 & 0.60 & 0.50 & 0.29 \\
\hline $\mathbf{4 2}$ & 0.65 & 0.55 & 0.53 & 0.35 & 0.45 & 0.47 \\
\hline $\mathbf{4 3}$ & 0.35 & 0.45 & 0.59 & 0.65 & 0.55 & 0.41 \\
\hline $\mathbf{4 4}$ & 0.40 & 0.35 & 0.35 & 0.60 & 0.65 & 0.65 \\
\hline $\mathbf{4 5}$ & 0.30 & 0.35 & 0.18 & 0.70 & 0.65 & 0.82 \\
\hline $\mathbf{4 6}$ & 0.20 & 0.10 & 0.29 & 0.80 & 0.90 & 0.71 \\
\hline $\mathbf{4 7}$ & 0.30 & 0.05 & 0.06 & 0.70 & 0.95 & 0.94 \\
\hline $\mathbf{4 8}$ & 0.00 & 0.15 & 0.06 & 1.00 & 0.85 & 0.94 \\
\hline $\mathbf{4 9}$ & 0.10 & 0.00 & 0.12 & 0.90 & 1.00 & 0.88 \\
\hline $\mathbf{5 0}$ & 0.15 & 0.05 & 0.06 & 0.85 & 0.95 & 0.94 \\
\hline $\mathbf{5 1}$ & 0.00 & 0.05 & 0.00 & 1.00 & 0.95 & 1.00 \\
\hline $\mathbf{5 2}$ & 0.00 & 0.00 & 0.06 & 1.00 & 1.00 & 0.94 \\
\hline & & & & & \\
\hline
\end{tabular}


Table.5 Length of growing period for each climatic condition

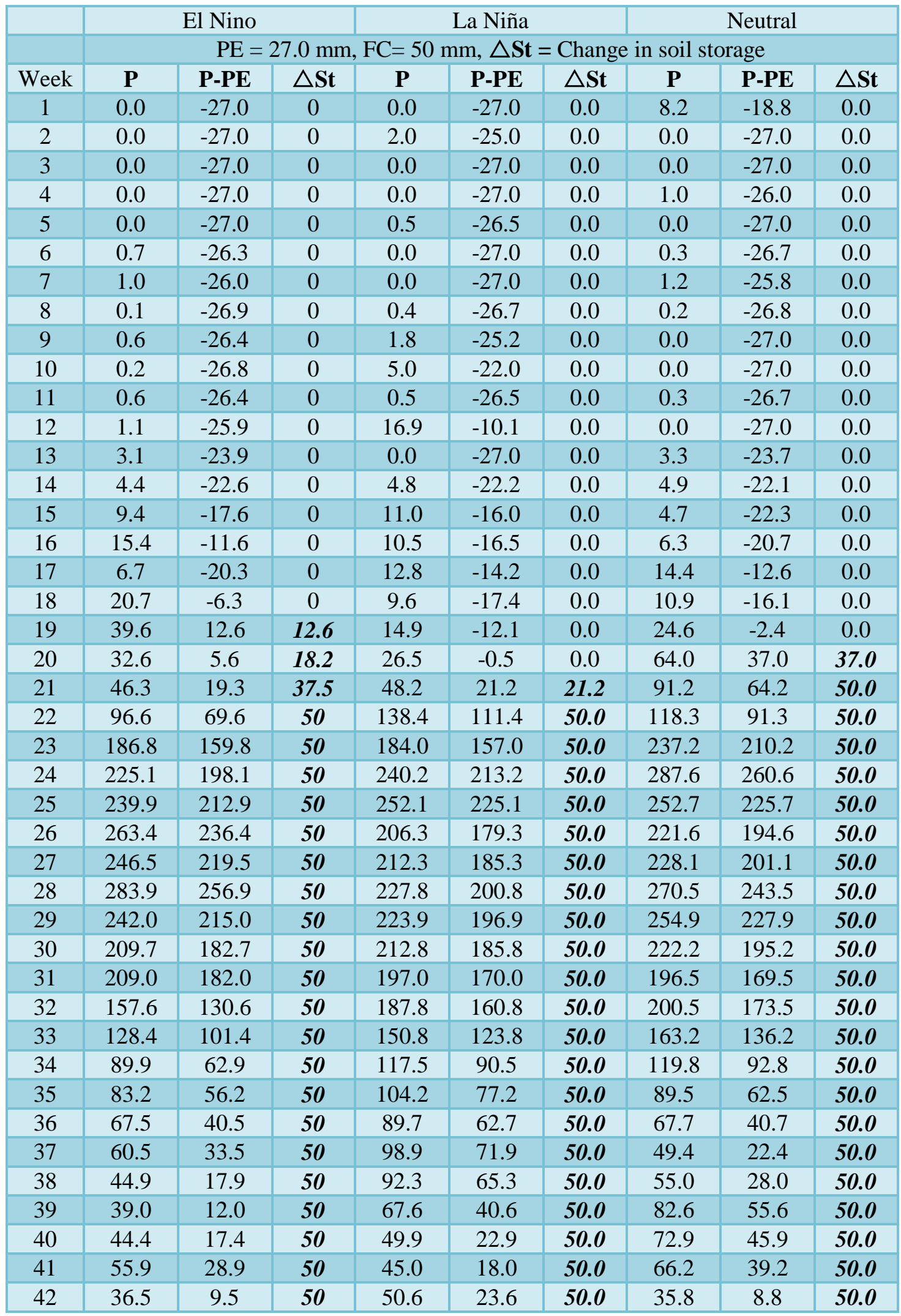




\begin{tabular}{|c|c|c|c|c|c|c|c|c|c|}
\hline 43 & 22.6 & -4.4 & 45.6 & 42.3 & 15.3 & 50.0 & 62.7 & 35.7 & 50.0 \\
\hline 44 & 36.1 & 9.1 & 50 & 26.3 & -0.7 & 49.3 & 38.1 & 11.1 & 50.0 \\
\hline 45 & 38.7 & 11.7 & 50 & 22.0 & -5.0 & 44.3 & 17.7 & -9.3 & 40.7 \\
\hline 46 & 21.7 & -5.3 & 44.7 & 7.2 & -19.8 & 24.5 & 19.5 & -7.5 & 33.2 \\
\hline 47 & 28.6 & 1.6 & 46.3 & 4.7 & -22.3 & 2.2 & 24.8 & -2.2 & 31 \\
\hline 48 & 1.1 & -25.9 & 20.4 & 13.2 & -13.8 & 0.0 & 9.4 & -17.6 & 13.4 \\
\hline 49 & 5.1 & -21.9 & 0 & 0.7 & -26.3 & 0.0 & 10.9 & -16.1 & 0.0 \\
\hline 50 & 10.6 & -16.4 & 0 & 4.9 & -22.1 & 0.0 & 6.8 & -20.2 & 0.0 \\
\hline 51 & 0.1 & -26.9 & 0 & 3.9 & -23.1 & 0.0 & 0.6 & -26.4 & 0.0 \\
\hline 52 & 0.7 & -26.3 & 0 & 2.2 & -24.8 & 0.0 & 3.0 & -24.0 & 0.0 \\
\hline LGP & \multicolumn{3}{|c|}{$\begin{array}{l}\text { El Nino } \\
\text { May)- } 49\left(09^{\text {th }} \text { Dec }\right) \\
31 \text { weeks }\end{array}$} & \multicolumn{3}{|c|}{$\begin{array}{l}\text { La Niña } \\
\text { May)- } 47\left(25^{\text {th }} \text { Nov }\right) \\
27 \text { weeks }\end{array}$} & \multicolumn{3}{|c|}{$\begin{array}{c}\text { Neutral } \\
20\left(14^{\text {th }} \text { May }\right)-49\left(09^{\text {th }} \text { Dec }\right) \\
29 \text { weeks }\end{array}$} \\
\hline
\end{tabular}

\section{Conditional probability}

The probability for continuous wet period can be seen in all the three situations during SW monsoon period. But during summer and NE monsoon period, El Niño conditions are having less probabilities for continuous wet spell, means probabilities are there for breakage of wet spells which may arise as a threat for rainfed cultivation during those periods.

\section{Frequency analysis}

From table 4 it can be inferred that the rainfall distribution is not at all affected during SW monsoon period for any of the climatic phenomenon. It is impacted much on the NW monsoon and summer periods. From both seasonal and annual distribution, it is evident that El Niño conditions increases the rainfall distribution to surplus for around 2-4 weeks in a year.

\section{Length of growing period}

The length of growing period was calculated for three climatic situations based on the method suggested by FAO, where the mean weekly precipitation exceeds the mean weekly evapotranspiration may be the wet weeks and period of continuous wet weeks along with stored soil moisture to meet the evapotranspiration requirement being the length of growing period (Table 5). It is inferred that since there is increased impact on NE monsoon, El nino conditions have better LGP (31 weeks) as compared with Lanina and neutral years. Even though LGP starts around the start of SW monsoon period; it ends at different stages during a year.

In conclusion apart from normal Indian conditions, even though the rainfall quantum is higher during the Lanina period, the distribution of rainfall is more during El Niño years, which enlighten the future possibilities of crop production in the area on behalf of the changing global climate scenarios.

\section{References}

Jeevananda Reddy, S. (1983). "Agro climatic classification of the Semi-Arid Tropics. A method for the computation of classificatory variables". Agricultural Meteorol., 30, 185-200.

Kingra, P.K., Gill, K.K and Singh, S. (2013). "Wet and Dry Spell Analysis for Crop Planning in Sub-Mountainous Punjab using Markov Chain Approach", J. Agrl. Phy., 13(2), 193-202

Krishnamurthy, K.N., Gowda, $\mathrm{M}$ and Vasanthkumar, T. (2015). "Markov Chain Model for Probability of weekly rainfall in Mandya district, Karnataka”, 
Intl. J. Innov. Res. Sci., 4,7, $6644-6650$. Selvaraju, R., Balasubramanian, T.N and Geethalakshmi, V. (1998). "On the variation of North east monsoon rainfall over Coimbatore during warm and cold phases of the El- Nino/Southern Osciallation (ENSO)", Vayu mandal, 50-53.
Veeraputhiran, R., Karthikeyan, R., Geethalakshmi, V., Selvaraju, R., Sundarsingh, S.D and Balasubramaniyan, T. N. (2003). "Crop planning - Climate Atlas, Manual”, A. E. publications, Coimbatore - 41 .

\section{How to cite this article:}

Rajesh Krishnan, R. K. and Retheesh, P. K. 2020. Fitting Crop Production in Northern Zone of Kerala during El Niño Conditions. Int.J.Curr.Microbiol.App.Sci. 9(11): 1469-1477.

doi: https://doi.org/10.20546/ijcmas.2020.911.173 